\title{
Interactions médicamenteuses
}

\author{
Une interaction médicamenteuse a lieu lorsque l'effet d'un médicament est aug- \\ menté ou diminué par l'administration concomitante ou parallèle d'un autre médi- \\ cament. Les patients prenant six médicaments ou plus ont une probabilité de $\mathbf{8 0} \%$ \\ de subir une interaction médicamenteuse et présentent donc un risque potentiel \\ d'effets indésirables.
}

Anne B. Taegtmeyer

Correspondance:

PD Dr Anne B. Taegtmeyer,

MRCP(UK), PhD

Klinische Pharmakologie und Toxikologie

Universitätsspital Basel

Hebelstrasse 2

CH-4031 Bâle

anne.taegtmeyer[at]usb.ch
Les patients âgés présentant plusieurs comorbidités ont une probabilité plus grande non seulement de subir une interaction médicamenteuse, mais aussi que cette interaction provoque un effet indésirable. De tels patients ont donc un risque accru de souffrir de complications du traitement.

\section{La distinction entre les mécanismes pharma- cologiques a une importance capitale}

On distingue deux mécanismes pharmacologiques différents à l'origine d'interactions. Les premiers sont d'ordre pharmacocinétique (modifications des concentrations de médicaments) et les seconds d'ordre pharmacodynamique (modifications de l'effet pharmacologique). Les interactions pharmacocinétiques peuvent être dues à une modification de la biodisponibilité ou de l'élimination (en anglais: «clairance»). Une réduction de la biodisponibilité orale survient par ex. lors de l'administration concomitante de cations polyvalents (calcium, magnésium, aluminium, fer) et de quinolones (par ex. ciprofloxacine), de lévothyroxine ou de tétracyclines (par ex. doxycycline). Les cations polyvalents forment de grands complexes (chélates) avec les médicaments chargés négativement. Ces complexes ne pouvant pas être résorbés par le système gastro-intestinal, la biodisponibilité du médicament diminue très fortement.

Les médicaments qui ont un «effet de premier passage» important sont ceux qui subissent déjà une forte élimination métabolique dans le tube digestif ou le foie. Ces médicaments ont donc aussi une biodisponibilité orale faible. En modifiant l'activité de la protéine de transport ou de l'enzyme impliquée dans le métabolisme, un autre médicament peut augmenter ou diminuer la biodisponibilité. En outre, les interactions pharmacocinétiques résultent non seulement d'une diminution ou d'une augmentation de l'élimination métabolique (hépatique) ou rénale (également par modification de l'activité de l'enzyme impliquée dans le métabolisme ou de la protéine de transport), mais aussi d'une modification de la liaison aux protéines.

Les enzymes «classiques» impliquées dans le métabolisme des médicaments appartiennent à la famille du cytochrome (CYP). Ce sont par exemple le CYP 3A4, le CYP 2D6 et le CYP 2C9. Les inhibiteurs «réputés» de ces enzymes sont notamment certains antibiotiques de la famille des macrolides (inhibiteurs du CYP 3A4, par ex. clarithromycine [Klacid $\left.{ }^{\oplus}\right]$ ), certains antifongiques azolés (inhibiteurs du CYP 3A4 et du CYP 2C9, par ex. fluconazole [Diflucan $\left.{ }^{\circledast}\right]$ ), certains inhibiteurs de la protéase (par ex. ritonavir [Norvir ${ }^{\circledR}$ ]) et certains inhibiteurs de la recapture de la sérotonine (inhibiteurs du CYP 2D6, par ex. fluoxétine [Fluctine $\left.{ }^{\circledast}\right]$ ). Les inducteurs «réputés» sont la rifampicine, la phénytoïne, la carbamazépine et le millepertuis. Des exemples d'interactions pharmacocinétiques figurent dans le tableau 1.

Des interactions pharmacodynamiques ont lieu lorsque les médicaments ont des effets biologiques identiques ou contraires. L'effet dépresseur central, additif et potentiellement dangereux des benzodiazépines utilisées en association avec de l'alcool en est un exemple classique. D'autres exemples figurent également dans le tableau 1.

\section{Reconnaître une interaction médicamenteuse}

Afin de pouvoir au mieux prévenir, diminuer ou surveiller dans la pratique clinique les conséquences éventuelles d'une interaction médicamenteuse, il peut s'avérer utile pour le médecin traitant d'évaluer quels sont les médicaments «agresseurs» et «victimes» dans l'interaction (tableau 2). Il faut toutefois

\section{Conseils pratiques}

1. Le recueil de l'anamnèse médicamenteuse complète est très important. Outre les médicaments soumis à ordonnance, les médicaments «over the counter» doivent être pris en compte, parce que ceux-ci peuvent également provoquer des interactions ayant de graves conséquences (par ex. préparations à base de millepertuis, de calcium).

2. La probabilité d'interactions augmente avec le nombre de médicaments pris. Il convient donc d'utiliser le moins de médicaments possible et pendant la durée la plus courte possible.

3. Les effets et, si cela est aussi possible et établi, les concentrations des médicaments doivent être surveillés.

4. Lorsque I'on prescrit un nouveau médicament, il faut rechercher d'éventuelles interactions avec la médication au long cours prise jusqu'ici. Les moyens électroniques («systèmes de détection des interactions») et les pharmacologues cliniques peuvent ici apporter une aide utile. 
Tableau 1

Interactions médicamenteuses importantes: une sélection personnelle. Pour les interactions avec les nouveaux anticoagulants oraux («NOACs»), l'auteure renvoit à Haschke M. Neue orale Antikoagulantien aus klinisch pharmakologischer Sicht. Ther Umsch. 2012;69:657-60.

\begin{tabular}{|c|c|c|c|}
\hline Médicament 1 & Médicament 2 & Effet & Mécanisme \\
\hline $\begin{array}{l}\text { Phenprocoumone } \\
\text { (Marcoumar }^{\circledast} \text { ) }\end{array}$ & $\begin{array}{l}\text { Anti-inflammatoires } \\
\text { non stéroïdiens }\end{array}$ & $\begin{array}{l}\uparrow \text { INR, } \\
\text { hémorragies } \\
\text { gastro- } \\
\text { intestinales }\end{array}$ & $\begin{array}{l}\text { Déplacement de la } \\
\text { phenprocoumone de sa } \\
\text { liaison aux protéines, lésions } \\
\text { gastro-intestinales, inhibition } \\
\text { plaquettaire }\end{array}$ \\
\hline $\begin{array}{l}\text { Anticoagulants oraux } \\
\text { ACO }\end{array}$ & $\begin{array}{l}\text { Inhibiteurs de l'agré- } \\
\text { gation plaquettaire }\end{array}$ & $\uparrow$ Hémorragies & $\begin{array}{l}\text { Interaction pharmaco- } \\
\text { dynamique }\end{array}$ \\
\hline $\begin{array}{l}\text { Phenprocoumone } \\
\text { (Marcoumar }{ }^{\circledast} \text { ) }\end{array}$ & $\begin{array}{l}\text { Inhibiteurs du CYP 3A4 } \\
\text { (clarithromycine, } \\
\text { fluconazole, voriconazole, } \\
\text { inhibiteurs de la protéase) } \\
\text { Inducteurs du CYP 3A4 } \\
\text { (phénytoïne, carbama- } \\
\text { zépine, rifampicine) }\end{array}$ & $\begin{array}{l}\uparrow \text { INR } \\
\downarrow \text { INR } \\
\end{array}$ & $\begin{array}{l}\downarrow \text { Métabolisme } \\
\uparrow \text { Métabolisme }\end{array}$ \\
\hline $\begin{array}{l}\text { Inhibiteurs sélectifs } \\
\text { de la recapture } \\
\text { de la sérotonine }\end{array}$ & 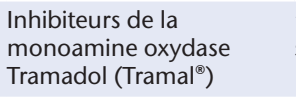 & $\begin{array}{l}\uparrow \text { Toxicité de la } \\
\text { sérotonine }\end{array}$ & $\begin{array}{l}\uparrow \text { Concentration de } \\
\text { sérotonine synaptique }\end{array}$ \\
\hline $\begin{array}{l}\text { Inhibiteurs de la calcineurine } \\
\text { (ciclosporine [Sandimmun }^{\oplus} \text { ] } \\
\text { et tacrolimus [Prograf }{ }^{\circledast} \text { ]) }\end{array}$ & $\begin{array}{l}\text { Inducteurs du CYP 3A4 } \\
\text { Inhibiteurs du CYP 3A4) }\end{array}$ & $\begin{array}{l}\uparrow \text { Toxicité } \\
\downarrow \text { Effets }\end{array}$ & $\begin{array}{l}\downarrow \text { Métabolisme } \\
\uparrow \text { Métabolisme }\end{array}$ \\
\hline Nitrates & $\begin{array}{l}\text { Inhibiteurs du PDE } \\
\left.\text { (sildénafil [Viagra }{ }^{\circledR}\right] \text { ) }\end{array}$ & Hypotension & $\begin{array}{l}\text { Inhibition de la dégradation } \\
\text { du GMPc } \rightarrow \text { Relaxation des } \\
\text { muscles lisses }\end{array}$ \\
\hline Lithium & $\begin{array}{l}\text { Anti-inflammatoires non } \\
\text { stéroïdiens }\end{array}$ & $\begin{array}{l}\text { Intoxication au } \\
\text { lithium }\end{array}$ & $\begin{array}{l}\downarrow \text { Filtration et } \\
\uparrow \text { réabsorption }\end{array}$ \\
\hline Digoxine & $\begin{array}{l}\text { Inhibiteurs de la } \\
\text { glycoprotéine P } \\
\text { (par ex. ciclosporine, } \\
\text { clarithromycine) }\end{array}$ & $\begin{array}{l}\uparrow \text { Effets de la } \\
\text { digoxine }\end{array}$ & $\begin{array}{l}\text { Inhibition des pompes } \\
\text { d'efflux } \\
\rightarrow \text { augmentation de } \\
\text { l'absorption de digoxine }\end{array}$ \\
\hline $\begin{array}{l}\text { Inhibiteurs de l'enzyme } \\
\text { de conversion de } \\
\text { l'angiotensine / antagonistes } \\
\text { des récepteurs de } \\
\text { l'angiotensine } 2\end{array}$ & $\begin{array}{l}\text { Suppléments potassiques } \\
\text { Diurétiques épargneurs } \\
\text { de potassium }\end{array}$ & Hyperkaliémie & $\begin{array}{l}\text { Effet pharmacodynamique } \\
\text { additif }\end{array}$ \\
\hline Atorvastatine, simvastatine & $\begin{array}{l}\text { Inhibiteurs du CYP 3A4, } \\
\text { par ex. clarithromycine }\end{array}$ & $\begin{array}{l}\uparrow \text { Risque de } \\
\text { myopathie }\end{array}$ & $\begin{array}{l}\downarrow \text { Captage hépatique } \\
\text { de la statine }\end{array}$ \\
\hline $\begin{array}{l}\text { 5-fluorouracile (5-FU), } \\
\text { flucytosine, capécitabine }\end{array}$ & Brivudine (Brivex ${ }^{\oplus}$ ) & $\begin{array}{l}\uparrow \text { Toxicité } \\
\text { du 5-FU }\end{array}$ & $\begin{array}{l}\text { Inhibition de la déhydro- } \\
\text { pyrimidine déshydrogénase } \\
\text { (DPD) }\end{array}$ \\
\hline $\begin{array}{l}\text { ACO, thyroxine, myco- } \\
\text { phénolate, œestrogènes, } \\
\text { vitamines ADEK }\end{array}$ & Cholestyramine & $\downarrow$ Effets & $\begin{array}{l}\text { Inhibition de la résorption, } \\
\text { interruption du cycle } \\
\text { entérohépatique (couma- } \\
\text { rine, mycophénolate mofétil) }\end{array}$ \\
\hline $\begin{array}{l}\text { Tétracyclines, quinolones, } \\
\text { lévothyroxine, lévodopa, } \\
\text { mycophénolate }\end{array}$ & Cations (Ca, Mg, Al, Fe) & $\downarrow$ Absorption & Formation de complexes \\
\hline Méthadone & $\begin{array}{l}\text { Autres médicaments } \\
\text { allongeant le QT }\end{array}$ & $\begin{array}{l}\text { Torsade de } \\
\text { pointes }\end{array}$ & $\begin{array}{l}\text { Allongement du QT additif } \\
\text { (effet pharmacodynamique) }\end{array}$ \\
\hline Méthotrexate & $\begin{array}{l}\text { Anti-inflammatoires } \\
\text { non stéroïdiens }\end{array}$ & Myélotoxicité & $\begin{array}{l}\downarrow \text { Filtration glomérulaire du } \\
\text { méthotrexate }\end{array}$ \\
\hline Tizanidine (Sirdalud ${ }^{\circledR}$ ) & Ciprofloxacine & $\begin{array}{l}\text { Chute de la } \\
\text { pression } \\
\text { artérielle, } \\
\text { somnolence }\end{array}$ & $\begin{array}{l}\downarrow \text { Métabolisme de la } \\
\text { tizanidine }\end{array}$ \\
\hline Azathioprine (Imurek ${ }^{\circledast}$ ) & Allopurinol (Zyloric ${ }^{\circledast}$ ) & Myélotoxicité & $\begin{array}{l}\text { Augmentation de la } \\
\text { formation de métabolites } \\
\text { toxiques par inhibition } \\
\text { de la xanthine oxydase }\end{array}$ \\
\hline
\end{tabular}

remarquer que cette répartition dépend de la situation et non du médicament. Certains médicaments sont des «victimes» dans une association, mais des «agresseurs» dans une autre association. L'administration concomitante de ciclosporine et de millepertuis provoque par exemple une chute dangereuse de
Tableau 2

Groupes de médicaments problématiques, répartis en «agresseurs» et «victimes».

\begin{tabular}{|c|c|}
\hline «Agresseurs» & «Victimes» \\
\hline Rifampicine, millepertuis & Anticoagulants oraux \\
\hline Antifongiques azolés & Antidiabétiques oraux \\
\hline $\begin{array}{l}\text { Antibiotiques de la famille des } \\
\text { macrolides }\end{array}$ & Statines \\
\hline Anti-inflammatoires non stéroïdiens & Antiépileptiques* \\
\hline Cations polyvalents & Immunosuppresseurs* \\
\hline
\end{tabular}

la concentration de ciclosporine. Dans cette situation, la ciclosporine doit être identifiée comme une «victime». L'administration concomitante de ciclosporine et de simvastatine provoque cependant une augmentation de la concentration de simvastatine (par inhibition du transport de la statine dans le foie, due à la ciclosporine) qui peut alors entraîner une toxicité musculaire. Dans cette situation, la ciclosporine joue le rôle «d'agresseur». Dans la pratique clinique quotidienne, il est également important de remarquer que les interactions médicamenteuses sont surtout pertinentes pour les médicaments à index thérapeutique faible. Tel est le cas notamment du Marcoumar (phenprocoumone), de la digoxine, de la ciclosporine déjà citée ((Sandimmun $\left.{ }^{\circledast}\right)$ et du lithium (Quilonorm ${ }^{\circledast}$ ).

Pour pouvoir distinguer les interactions sans conséquences de celles susceptibles d'avoir de graves répercussions, une compréhension de la pharmacologie et de la médecine est nécessaire. Des moyens électroniques («systèmes de détection des interactions») sont aussi disponibles de nos jours. Mais les résultats peuvent être volumineux et ne pas être toujours cliniquement significatifs. Ils ne sont donc pas complètement applicables dans la pratique quotidienne clinique $[1,2]$.

1 Taegtmeyer et al. Clinical usefulness of electronic drugdrug interaction checking in the care of cardiovascular surgery inpatients. Cardiology 2012;123:219-22

2 Beeler PE et al. Use of an on-demand drug-drug interaction checker by prescribers and consultants: a retrospective analysis in a Swiss teaching hospital. Drug Saf. 2013;36:427-34.

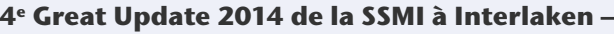 une formation continue durable dans un cadre source d'inspiration}

Pour la quatrième fois, la Société Suisse de Médecine Interne Générale (SSMI) organise un Great Update les $\mathbf{2 5}$ et $\mathbf{2 6}$ septembre au centre des congrès Kursaal d'Interlaken. De nombreux intervenants de haut rang ont élaboré un programme passionnant axé sur la pratique, notamment l'auteure de cet article qui s'est intéressée aux «Interactions médicamenteuses». Une grande partie des participants vient de Suisse romande. Vous trouverez plus d'informations sur le Great Update 2014 sous www.imk.ch/greatupdate2014 